\title{
BudgetMITverantwortung und KVG-Revision Managed Care / Integrierte Versorgung
}

\author{
Der Schweizer Dachverband der Ärztenetze «medswiss.net» hat als Erstverfasser \\ den Begriff «Budgetmitverantwortung» geprägt und legt nun eine präzise Definition \\ vor.
}

Die Gesetzesvorlage zur Integrierten Versorgung (Managed Care) will den Versicherten einen reduzierten Selbstbehalt gewähren, wenn sie ein Krankenversicherungsmodell wählen, das eine Budgetmitverantwortung der Leistungserbringer vorsieht.

Der Begriff «Budgetmitverantwortung» wurde 2005 von einer Arbeitsgruppe des Schweizer Dachverbandes der Ärztenetze «medswiss.net» geschaffen im Rahmen der Ärztenetzwerk-Definition «Vision 08» und hat sich seither im Sprachgebrauch etabliert. medswiss.net wollte darauf hinweisen, dass die Managed-Care-Verträge die ökonomische Verantwortung nie ausschliesslich den Ärztenetzen übertragen haben, sondern dass diese Verantwortung von beiden Vertragspartnern, Versicherern und Ärztenetz, gemeinsam getragen wird.

Die vorliegende Definition von medswiss.net (siehe Kasten) berücksichtigt Erkenntnisse aus 15-jähriger Erfahrung mit Managed Care in der Schweiz.

Zentrale Elemente ökonomischer Verantwortung sind Kollektiv, Budget und Gewinn-Verlust-Beteiligung.

\section{Definition des Begriffs «Budgetmitverantwortung» \\ - Budgetmitverantwortung ist eine zwischen den Managed-Care-Vertragspartnern ausge- handelte und gemeinsam getragene ökono- mische Verantwortung für die Kosten der obligatorischen Krankenpflegeversicherung eines Kollektives von Managed-Care-Versicher- ten. \\ - Vertragspartner sind einerseits Versicherer und andrerseits ein Kollektiv von Leistungser- bringern. \\ - Die Vertragspartner handeln eine Leistungs- vereinbarung aus (Managed-Care-Vertrag / Vertrag der Integrierten Versorgung). \\ - Die Budgetmitverantwortung ist ein verhan- delbarer Bestandteil dieses Vertrages und um- fasst das Budget und die Gewinn-Verlust-Be- teiligung. \\ - Das Budget für das versicherte Kollektiv muss risikogerecht berechnet werden. Die Risiko-}

Korrespondenz:
Dr. med. Jörg Fritsch Kleinbuholz 3 CH-6012 Obernau

j.fritschi@hin.ch

\section{Das Kollektiv}

Managed Care bzw. Integrierte Versorgung kann nur über das Kollektiv der Versicherten und der medizinischen Leistungsträger verantwortungsbewusst umgesetzt werden. Die Leistungsträger organisieren sich dafür in einer geeigneten Gesellschaftsform (z. B. Verein, Aktiengesellschaft).

\section{Das Budget}

Ein Budget ist eine verhandelbare Kostenzielvorgabe für die Betreuung eines Versichertenkollektives. Verhandlungselemente sind der Budgetbetrag, die Budgetberechnungsmethode, Budgetumfang und Budgetaktivität.

\section{Budgetberechnungsmethoden}

Übliche Budgetberechnungsmethoden sind:

- Vergleich mit einem konventionell versichertem Kollektiv (Parallelkostenrechnung);

- Vergleich mit den Vorjahreskosten unter Berücksichtigung der Teuerung (Vorjahreskostenrechnung);

indikatoren können vertraglich festgelegt werden (Personendaten, geeignete Morbiditätsindikatoren', versicherungstechnische Faktoren ${ }^{2}$ ).

- Die Modalitäten der Gewinn-Verlust-Beteiligung werden von den Vertragspartnern vereinbart.

- Budget und Budgetmitverantwortung gelten nur für Kollektive. Das Budget bezieht sich auf das Kollektiv der Versicherten. Die Budgetmitverantwortung wird vom Kollektiv der Leistungserbringer getragen.

(Erarbeitet durch die im Dachverband vereinigten Ärztenetze)

\footnotetext{
z.B. Vorhospitalisationen, pharmaceutical cost groups

2 Höhe der Franchise des Versicherten, Einsparziele, Grossrisikoschwellenwerte usw.
} 
- Capitation oder Kopfpauschale als prospektive Budgetberechnungsmethode aufgrund individueller, krankheitsbasierter Versichertendaten. Auch Gesunde tragen zum Budget bei, womit sich die Prävention besonders lohnt.

Merke: Capitation ist lediglich ein Instrument der Budgetberechnung und schliesst nicht automatisch eine ökonomische Mitverantwortung ein.

\section{Budgetumfang}

Das Budget kann die ganze Behandlungskette oder nur einen Teil davon umfassen. Verträge über die ganze Behandlungskette sind vorzuziehen. Damit entfallen Anreize zur Risikoverlagerung in andere Sektoren des Gesundheitswesens.

Ärztenetze sind bereit, auch chronisch Kranke in die integrierte Versorgung einzubinden. Voraussetzung ist eine morbiditätsgerechte Budgetberechnung.

\section{Budgetaktivität}

Ohne Gewinn-Verlust-Beteiligung bleibt ein Budget informell. Dies entspricht dem «einfachen» Hausarztvertrag als niederschwelligem Einstiegsmodell für junge Ärztenetze in die Integrierte Versorgung. Erst die Beteiligung am Gewinn oder Verlust (Risikobeteiligung*) stellt das Budget aktiv und bedeutet ökonomische Mitverantwortung.

\section{Die Gewinn-Verlust-Beteiligung}

Die Beteiligung an Gewinn oder Verlust ist das zentrale Element ökonomischer Mitverantwortung. Sie ist ebenfalls verhandelbar. Der budgetierte Betrag wird dem Ärztenetz nicht ausbezahlt, bleibt also virtuell. Der Budgetbetrag ist nicht eine Leistungsvergütung.

Der Budgetbetrag und die tatsächlichen Kosten werden verglichen; damit wird ein Gewinn oder ein Verlust sichtbar. Der Leistungsvertrag zwischen Versicherer und Ärztenetz bestimmt die Form und das Mass der Beteiligung. Üblich ist eine betragliche Beteiligung als Bonus/Malus oder eine prozentuale Beteiligung.

Für die Gewinn-Verlust-Beteiligung haftet nur das Ärztenetz, nie jedoch seine einzelnen Mitglieder.

Weitere Sicherungsmassnahmen können vorgesehen werden: Limitationen, Rückstellungen, Rückversicherungen, Grossrisikoabsicherung.

Eine vollständige ökonomische Verantwortung läge vor, wenn das Risiko im Sinne eines Globalbudgets vollumfänglich vom Ärztenetz getragen wird. Das Ärztenetz übernähme damit die Rolle des Versicherers.
Der Schweizer Dachverband der Ärztenetze befürwortet seit jeher eine Trennung von Kosten- und Leistungsträgern. Assekuranz und Medizin verlangen je eigene Kernkompetenzen. Werden beide Bereiche zusammengelegt, würde die Gesundheitsversorgung finanziell durch ein Globalbudget gesteuert.

Ist die Versorgungssicherheit auch im Rahmen einer Budgetmitverantwortung gewährleistet? Eindeutig ja!

Budgetmitverantwortung garantiert sogar Versorgungssicherheit. Die Lösung heisst Versorgungsausgleich. In der konventionellen Krankenversicherung werden die Leistungen einzeln verrechnet. Damit besteht ein Anreiz zur auch nicht immer ungefährlichen Überversorgung. Auf der anderen Seite würde ein alleiniges Budget zur Unterversorgung tendieren.

In einem Ärztenetz mit Budgetmitverantwortung rechnen die Ärztinnen und Ärzte gemäss Einzelleistungstarif ab. Gleichzeitig sind sie dem virtuellen Budget des Netzes verpflichtet. Dadurch werden die beiden Tendenzen Überversorgung/Unterversorgung ausgeglichen. Versorgungsausgleich bedeutet Versorgungssicherheit.

\section{Gesetzliche Vorschriften?}

Wie soll bzw. kann Managed Care gesetzlich vorgeschrieben werden?

Managed Care im Sinne der integrierten, gesteuerten Gesundheitsversorgung ist mehr als ein Produkt. Managed Care basiert auf der Bereitschaft aller Beteiligten zur Zusammenarbeit. Zusammenarbeit setzt Vertrauen voraus. Rigide Vorschriften können dazu führen, dass Managed-Care-Verträge nicht abgeschlossen werden. Gefordert sind gute gesetzliche Rahmenbedingungen, die Managed Care fördern.

Verband der Schweizer Ärztenetze
medswiss.net
Der Verband umfasst 47 Hausarztvereine und
Ärztenetze mit rund 2000 Ärztinnen und Ärzten,
die etwa 600000 Managed-Care-Versicherte be-
treuen. Als Kollektive mit verbindlichen Stan-
dards sind Ärztenetze ganz besonders fähig, die
vom KVG geforderten Leistungskriterien Wirk-
samkeit, Zweckmässigkeit, Wirtschaftlichkeit und
Qualität zu leben und abzubilden und zusam-
men mit interessierten Versicherern eine be-
darfsgerechte Gesundheitsversorgung anzubie-
ten.

